www.nature.com/ejhg

\title{
Apolipoprotein E gene in frontotemporal dementia: an association study and meta-analysis
}

\author{
Patrice Verpillat ${ }^{*, 1,2}$, Agnès Camuzat ${ }^{3}$, Didier Hannequin ${ }^{4,5}$, \\ Catherine Thomas-Anterion ${ }^{6}$, Michèle Puel ${ }^{7}$, Serge Belliard ${ }^{8}$, Bruno Dubois ${ }^{9}$, \\ Mira Didic $^{10,11}$, Lucette Lacomblez ${ }^{12,13}$, Olivier Moreaud ${ }^{14}$, Véronique Golfier ${ }^{8}$, \\ Dominique Campion ${ }^{4}$, Alexis Brice ${ }^{3,9,15}$ and Françoise Clerget-Darpoux ${ }^{1}$
}

\footnotetext{
${ }^{1}$ INSERM U535, Le Kremlin Bicêtre, France; ${ }^{2}$ Département d'Epidémiologie, de Biostatistique et de Recherche Clinique, Centre Hospitalier Universitaire Bichat-Claude Bernard, AP-HP/Université Paris VII, Paris, France; ${ }^{3}$ INSERM U289, Centre Hospitalier Universitaire Pitié-Salpêtrière, Paris, France; ${ }^{4}$ INSERM EPI 9906, Rouen, France; ${ }^{5}$ Service de Neurologie, Centre Hospitalier Universitaire, Rouen, France; ${ }^{6}$ Service de Neurologie, Centre Hospitalier Universitaire, Saint-Etienne, France; ${ }^{7}$ Service de Neurologie, Centre Hospitalier Universitaire Purpan, Toulouse, France; ${ }^{8}$ Service de Neurologie, Centre Hospitalier Universitaire Pontchaillou, Rennes, France; ${ }^{9}$ Fédération de Neurologie, Centre Hospitalier Universitaire Pitié-Salpêtrière, Paris, France; ${ }^{10}$ Service de Neurologie et Neuropsychologie, Centre Hospitalier Universitaire de la Timone, Marseille, France; ${ }^{11}$ INSERM EMI U9926, Marseille, France; ${ }^{12}$ Fédération de Neurologie Mazarin, Centre Hospitalier Universitaire Pitié-Salpêtrière, Paris, France; ${ }^{13}$ Département de Pharmacologie, Centre Hospitalier Universitaire Pitié-Salpêtrière, Paris, France; ${ }^{14}$ Service de Neurologie, Centre Hospitalier Universitaire, Grenoble, France; ${ }^{15}$ Département de Génétique, Cytogénétique et Embryologie, Centre Hospitalier Universitaire Pitié-Salpêtrière, Paris, France
}

No definite genetic risk factor of non-monogenic frontotemporal dementia (FTD) has yet been identified. Several groups have examined the potential association of FTD with the apolipoprotein E (APOE) gene, but the results are inconsistent. Our objective was to determine whether APOE is a risk factor of FTD, using the largest series of patients with FTD and controls analysed so far (94 unrelated patients and 392 age and sex-matched controls), and a meta-analysis. Homozygosity for the E2E2 genotype was significantly associated with FTD (odds ratio $(O R)=11.3 ; P=0.033$, exact test). After stratification on familial history (FH) for FTD, the OR for E2E2 was still found significant when analysing only patients with a positive $\mathrm{FH}(\mathrm{OR}=\mathbf{2 3 . 8} ; P=\mathbf{0 . 0 1 9})$. The meta-analysis, using 10 case-control studies with available genotype or allele information, comprising a total of 364 FTD patients and 2671 controls, including the patients of the present study, did not reach statistical significance even if the E2E2 genotype was more frequent in patients than in controls ( 0.018 vs 0.006 , respectively). Because of studies heterogeneity (Mantel-Haenszel statistics: $P=0.004$ ), we analysed on one hand the neuropathologically-confirmed studies, and on the other hand the clinical-based studies. In the neuropathologically-confirmed studies (Mantel-Haenszel statistics: $P=n s$ ), we found a significant increase of the E2 allele frequency in FTD patients (OR[E2 vs $\mathrm{E} 3]=2.01 ; 95 \% \mathrm{Cl}=1.02-3.98 ; P=0.04)$. The same result was found in the clinical-based studies, but studies heterogeneity remained. No result was significant with the E4 allele. The E2 allele

\footnotetext{
*Correspondence: Dr Patrice Verpillat; Hôpital Bichat, Département d'Epidémiologie, de Biostatistiques et de Recherche Clinique, 46 rue Henri Huchard, 75877 Paris cedex 18, France. Tel: +33 1402562 55; Fax: +331402567 73; E-mail: patrice.verpillat@bch.ap-hop-paris.fr Received 21 December 2001; revised 15 April 2002; accepted 18 April 2002
} 
seems so to be a risk factor of FTD whereas this allele is associated with the lowest risk in Alzheimer's disease. If this finding was confirmed, it could provide new insights into the mechanisms of differential risk related to APOE in neurodegenerative diseases. European Journal of Human Genetics (2002) 10, 399-405. doi:10.1038/sj.ejhg.5200820

Keywords: apolipoprotein E gene; frontotemporal dementia; genetic association; meta-analysis

\section{Introduction}

Frontotemporal dementia (FTD) is one of the most common cause of neurodegenerative dementia after Alzheimer's disease (AD). ${ }^{1}$ Since the Lund and Manchester groups consensus conference in April $1994,{ }^{2}$ clinical and neuropathological criteria for FTD have been well established. This has lead to a better distinction of FTD from AD, which is the most frequent misdiagnosis of FTD. ${ }^{3,4}$ Recently, mutations in the tau gene localised to $17 q 21$ have been identified in several families with autosomal dominant inheritance. $^{5-8}$ To date, ten missense mutations, two deletions, and three transition mutations not altering the encoded amino-acid sequence have been identified in exons of the tau gene. ${ }^{9}$ In addition, six intronic mutations have also been found in the $5^{\prime}$ splice donor site of exon $10 .^{9,10}$ These mutations were detected in 25 to $40 \%$ of families with FTD that have autosomal dominant inheritance. ${ }^{6,8,11}$ However, genetic heterogeneity has been demonstrated, since another locus for FTD has been mapped on chromosome 3 in a single large pedigree. $^{12}$ The genetics of non-monogenic FTD has been less studied, although several groups have examined the potential association of FTD with the apolipoprotein E (APOE) gene, with inconsistent results. ${ }^{13-26}$

The E4 allele of the APOE gene is now undeniably the most important risk factor in non-monogenic forms of AD. Although findings have not been as consistent as in $\mathrm{AD}$, an increase in the APOE E4 allele frequency has been variably observed in other forms of dementia, including diffuse Lewy body disease ${ }^{27-29}$ and vascular dementia. ${ }^{30,31}$ But it does not appear to be an important risk factor in several other neurodegenerative processes, like progressive supranuclear palsy $^{32}$ and Parkinson's disease. ${ }^{33,34}$ Some reports have suggested that APOE might also be a risk factor in FTD. Initially, Gustafson et $a l^{13}$ reported a higher E4 allele and E4E4 genotype frequency in patients with FTD than in controls, and an even more significant increase of the E2 allele. Stevens et $a l^{14}$ have also found a significantly increased risk for the E4 allele and E4E4 genotype in FTD. Subsequently, the results of a further study have suggested that the E2 allele, rather than the E4 allele, could be the risk factor for FTD. ${ }^{15}$ Finally, a recent study has shown a significant increase of the E4 allele in an FTD population compared to healthy controls. ${ }^{26}$ But ten other studies have not replicated this association. ${ }^{16-25}$

Because all previous studies have been based on relatively small samples (less than 35 except one study with
88 FTD patients), we have re-examined the role of APOE in a larger case-control study of 94 FTD patients and 392 controls. We performed also a meta-analysis using all published findings of similar case-control studies conducted until November 2001 to evaluate the effect of APOE as a risk factor for FTD.

\section{Methods}

\section{Subjects}

All patients were ascertained over a 3-year period through consecutive admissions to 10 university hospitals in France. In this sample, we analysed the tau gene in probands with autosomal dominant inheritance by direct sequencing (exons 1-5, 7 and 9-14, and the corresponding flanking intronic sequences). The same mutation, P301L, was found in six patients. ${ }^{8}$ After excluding these six patients with autosomal dominant inheritance and mutation of tau, our sample for the present study was thus composed of 94 unrelated patients with FTD (41.5\% men). All patients with FTD underwent a thorough clinical examination, including personal and familial medical history, neurological and psychiatric investigations, psychometric testing (MiniMental State Examination ${ }^{35}$, Mattis Dementia Rating Scale $^{36}$, Verbal Learning Test $^{37}$ and Frontal Assessment Battery $^{38}$ ), laboratory tests, computed tomography and magnetic resonance imaging, regional cerebral blood flow measurement (single-photon emission computed tomography) and electroencephalography. The diagnosis of FTD was established according to the Lund-Manchester clinical consensus criteria for FTD. $^{2}$ Age at onset was assessed by interviewing a next of kin and was defined as the age at which relevant symptoms first appeared according to the family (mean $\pm S D$ age at onset, $60.6 \pm 8.5$ years; range, $43-77$ years). In our study, we identified 31 patients with one or more first- or second-degree relatives with FTD (33\%; 35.5\% men; mean \pm SD age at onset, 60.2 \pm 7.9 years; range, 45-77 years), but without clear autosomal dominant inheritance and without mutation in the tau gene.

Patients with FTD were compared with 392 age- and sexmatched controls (44.9\% men; mean \pm SD age at examination, $62.5 \pm 8.7$ years; range, $38-77$ years). Control subjects were the patients' spouses or healthy blood donors or individuals living in nursing homes. None of the controls showed cognitive deficits or behavioural disturbance. 
All participants in this study were white and were living in France. Informed written consent was obtained for all participants, either directly or from the legal tutor.

\section{Meta-analysis}

For the meta-analysis we searched through Medline for all studies performed for APOE in FTD until November 2001, and reviewed the references in the retrieved articles. Fourteen association studies were found. ${ }^{13-26}$ Two different meta-analysis were performed: one with the genotypes, and another with the alleles. For the first meta-analysis, we included only the seven studies with available information on exact genotype frequencies. ${ }^{14,15,18,20,22-24}$ For the second meta-analysis, three additional studies with available information on allele frequencies only were included. ${ }^{13,17,21}$ Four studies could not be included in any meta-analysis because no information on exact genotype or allele frequencies was provided. ${ }^{16,19,25,26}$ In all selected studies, we have verified that the controls were in HardyWeinberg Equilibrium (HWE). Table 1 provides the clinical descriptions and design of the studies included in the meta-analysis.

\section{Genetic analysis}

DNA was extracted from blood lymphocytes using standard methods. APOE genotypes were determined by restriction enzyme digestion according to the method of Hixson and Vernier. ${ }^{39}$

\section{Statistical analysis}

Power computations were made with nQuery Advisor ${ }^{\circledR}$ Release 4.0. Statistical analysis were performed using SAS software release 8.0 (SAS Institute, Cary, NC, USA). For initial comparisons, the two-sided $\chi^{2}$ test or Fisher exact test when appropriate was used to determine potential differences in the distribution of the APOE genotypes and alleles between groups. Student $t$-test was employed to compare differences between mean age at onset and mean age at examination in the two groups, and non parametric Mann-Whitney test to compare differences between mean age at onset in the group with FTD according to APOE genotypes. Mantel-Haenszel-Peto statistics was performed to test for heterogeneity between studies selected for the meta-analysis and to compute weighted odds ratio. ${ }^{40,41}$

\section{Results}

\section{Association study}

No deviation from the HWE was observed among controls. Assuming a two-sided significance level $(\alpha)$ of 0.05 and a power $(1-\beta)$ of $80 \%$, the size of our sample was sufficient to detect an OR of at least 1.8 when bearing the E4 allele and 2.2 when bearing the E2 allele.

Genotype and allele frequencies in the control group were similar to frequencies reported in Caucasian populations (Table 2). The distribution of the APOE genotypes among patients with FTD and controls was significantly different $(P=0.041$, exact test). We found that the E2E2 frequency was more than 10 -fold higher in patients than

Table 2 APOE genotype and allele frequencies in patients with FTD and controls

\begin{tabular}{llll}
\hline & FTD patients & Controls & \\
\hline Genotypes & $(n=94)$ & $(n=392)$ & \\
E2E2 & $3.2(3)$ & $0.3(1)$ & \\
E2E3 & $13.8(13)$ & $13.0(51)$ & \\
E2E4 & $2.1(2)$ & $3.3(13)$ & \\
E3E3 & $63.8(60)$ & $57.6(226)$ & \\
E3E4 & $14.9(14)$ & $24.5(96)$ & \\
E4E4 & $2.1(2)$ & $1.3(5)$ & $P=0.041$ (exact test) \\
Alleles & $(n=188)$ & $(n=784)$ & \\
E2 & $11.2(21)$ & $8.4(66)$ & \\
E3 & $78.2(147)$ & $76.4(599)$ & \\
E4 & $10.6(20)$ & $15.2(119)$ & $P=0.171$ \\
\hline
\end{tabular}

Table 1 Clinical description of the populations included in the meta-analysis

\begin{tabular}{|c|c|c|c|c|c|c|c|c|c|c|c|c|}
\hline \multirow[b]{2}{*}{ Study } & \multirow[b]{2}{*}{$\begin{array}{l}\text { Country } \\
\text { (region) }\end{array}$} & \multirow[b]{2}{*}{$\mathrm{n}$} & \multicolumn{6}{|c|}{ Case samples } & \multicolumn{4}{|c|}{ Control samples } \\
\hline & & & $\begin{array}{l}\text { Sample } \\
\text { type }\end{array}$ & $\begin{array}{l}\% \\
\mathrm{FH}+\end{array}$ & $\begin{array}{l}\% \\
M\end{array}$ & $\begin{array}{l}\text { Age, years } \\
\text { mean } \pm S D\end{array}$ & $\begin{array}{l}\text { Diagnostic } \\
\text { criteria }\end{array}$ & $\begin{array}{l}\% \\
N P\end{array}$ & $\mathrm{n}$ & $\begin{array}{l}\text { Clinical } \\
\text { status }\end{array}$ & $\%$ & $\begin{array}{l}\text { Age, years } \\
\text { mean } \pm S D\end{array}$ \\
\hline Farrer et al. ${ }^{22}$ & USA (MA) & 10 & $\mathrm{C}$ & 58 & 100 & $60.4 \pm 5.4^{*}$ & $A C$ & 100 & 1204 & - & - & $\S-1$ \\
\hline Geschwind et al. ${ }^{20}$ & USA (CA) & 33 & C & 42 & 48 & $55 \pm \overline{11}$ * & $\mathrm{L}-\mathrm{M} / \mathrm{AC}$ & 33 & 30 & $\mathrm{AC}$ & 43 & $73 \pm 14$ \\
\hline Gomez-Isla et al. ${ }^{24}$ & USA (MA) & 31 & C & - & 52 & $68 . \overline{3} \pm 13.3 \dagger$ & $A C$ & 100 & 129 & - & 40 & $77 . \overline{8} \pm 13.5$ \\
\hline Gustafson et al. ${ }^{13}$ & Sweden & 21 & C & 52 & 29 & $59 \pm 10.5^{\star}$ & L-M & 0 & 29 & C & - & $\S-2$ \\
\hline Helisalmi et al. ${ }^{18}$ & Finland & 9 & C & - & 33 & $66 \pm 8^{*}$ & - & - & 60 & - & 47 & $69 \pm 8$ \\
\hline Lehmann et al. ${ }^{15}$ & UK (Oxford) & 11 & $P$ & 0 & 45 & $65 . \overline{7} \div$ & L-M/AC & 82 & 136 & C & 43 & $77 . \overline{5}$ \\
\hline Pickering-Brown et al. ${ }^{17}$ & UK (Manchester) & 27 & C & - & - & - & L-M/AC & 48 & 35 & - & - & - \\
\hline Pickering-Brown et al. ${ }^{21}$ & UK (Manchester) & 88 & C & 34 & 53 & $56.9 \pm 10.1^{*}$ & L-M/AC & 56 & 35 & - & - & - \\
\hline Schneider et $a .^{23}$ & USA (GA) & 6 & C & - & - & - & $\mathrm{AC}$ & 100 & 95 & - & - & - \\
\hline Stevens et al. ${ }^{14}$ & The Netherlands & 34 & $\mathrm{P}$ & 0 & 35 & $52.1 \pm 9.2^{*}$ & L-M & 0 & 561 & $\mathrm{C}$ & 43 & $59.9 \pm 2.8$ \\
\hline Current study & France & 94 & C & 33 & 41 & $60.6 \pm 8.5^{*}$ & $\mathrm{~L}-\mathrm{M} / \mathrm{AC}$ & 3 & 392 & C & 45 & $62.5 \pm 8.7$ \\
\hline
\end{tabular}

*Age at onset; †age (not specified); fage at death or last examination; §only the range of age is specified (for $1: 22$ to 71 , for $2: 25$ to 50 ). $\mathrm{FH}+=$ positive familial history; $\% \mathrm{M}=\%$ of males; $\% \mathrm{NP}=\%$ of neuropathological confirmation; C=clinical-based sample; $\mathrm{P}=\mathrm{population-based}$ sample; $A C=$ autopsy confirmed; L-M=Lund-Manchester clinical consensus criteria for FTD ${ }^{2} ;-=$ not available. 
in controls (OR[E2E2 vs E3E3]=11.3; $P=0.033$, exact test). Heterozygosity did not involve a significantly increased risk. The allele distribution was not significantly different between patients and controls, even if we observed a nonsignificant increase of the E2 allele frequency in patients. After stratification on the absence $(\mathrm{FH}-)$ or presence $(\mathrm{FH}+)$ of familial history of FTD, the result was even more significant in the group with a positive familial history $(\mathrm{FH}+)$ of FTD (E2E2 vs E3E3: in $\mathrm{FH}+$ group, $\mathrm{OR}=23.8$; $P=0.019$, exact test; in $\mathrm{FH}-$ group, $\mathrm{OR}=5.5 ; P=0.29$, exact test). This result remained significant even after a Bonferroni correction for multiple testing (corrected $P$-value $=0.038$ ). Further analysis of the APOE genotype in the whole sample of patients with FTD showed that the three patients with the E2E2 genotype had a lower mean age at onset than the 91 patients lacking this genotype $(54.7 \pm 8.4$ and $60.7 \pm 8.4$, respectively), but this difference was not statistically significant, probably because of the small sample size. The same tendency was found in the $\mathrm{FH}+$ group, but not in the $\mathrm{FH}$ - group (data not shown).

The same analyses were performed with the E3E4 and E4E4 genotypes, and the E4 allele. None was significant.

\section{Meta-analysis}

For the meta-analysis of the APOE polymorphism in FTD, we were able to pool seven case-control studies with genotype information (total of 228 FTD patients and 2607 controls including ours), ${ }^{14,15,18,20,22-24}$ and three more with allele information (total of 364 FTD patients and 2671 controls including ours). ${ }^{13,17,21}$ Some studies included only neuropathologically-confirmed patients, ${ }^{22-24}$ when others were mainly clinical-based, ${ }^{13-15,17,18,20,21}$ as ours. All populations were Caucasian.

We have first used the studies with available information on the genotype distribution among patients with FTD and controls. There was no significant difference between patients and controls (Table 3). The E2E2 genotype was more frequent in patients than in controls but this difference failed to reach statistical significance (OR[E2E2 vs E3E3]=3.08; $P=0.06$, exact test). We next investigated the association with the E2 allele in the 10 selected studies (Table 3). We found a significant increase of the E2 allele frequency in patients with FTD compared to controls (OR[E2 vs E3]=1.64; $P=0.004$ ) (Figure 1). But the MantelHaenszel statistics to test heterogeneity among studies was significant $(P=0.004)$. Instead of considering one or more of the data sets as an outlier based on APOE genotype distribution and excluding it or them from the meta-analysis, we decided to reduce the heterogeneity of the meta-analysis in discriminating the studies according to the mode of recruitment (autopsy or clinic). Considering the 10 studies selected for the meta-analysis, three ${ }^{22-24}$ were based only on neuropathologically-confirmed FTD patients, and another $^{21}$ provided allelic information on a subgroup of autopsied patients. For the latter, we have so added this
Table 3 Meta-analysis of APOE distribution in patients with FTD and controls

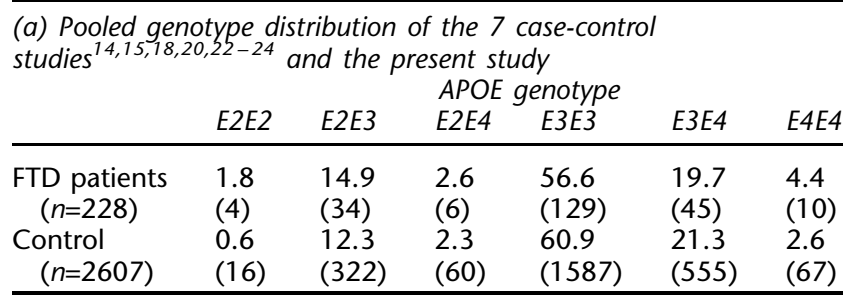

$P=\mathrm{ns}$

(b) Pooled allele distribution of the 10 case-control studies $^{13-15,17,18,20-24}$ and the present study

\begin{tabular}{llll} 
& \multicolumn{3}{c}{ APOE allele } \\
& E2 & E3 & E4 \\
\hline FTD patients $(n=364)$ & $10.2(74)$ & $73.1(532)$ & $16.7(122)$ \\
Controls $(n=2671)$ & $7.8(419)$ & $77.8(4155)$ & $14.4(768)$ \\
\hline$P=0.014$.
\end{tabular}

$P=0.014$.

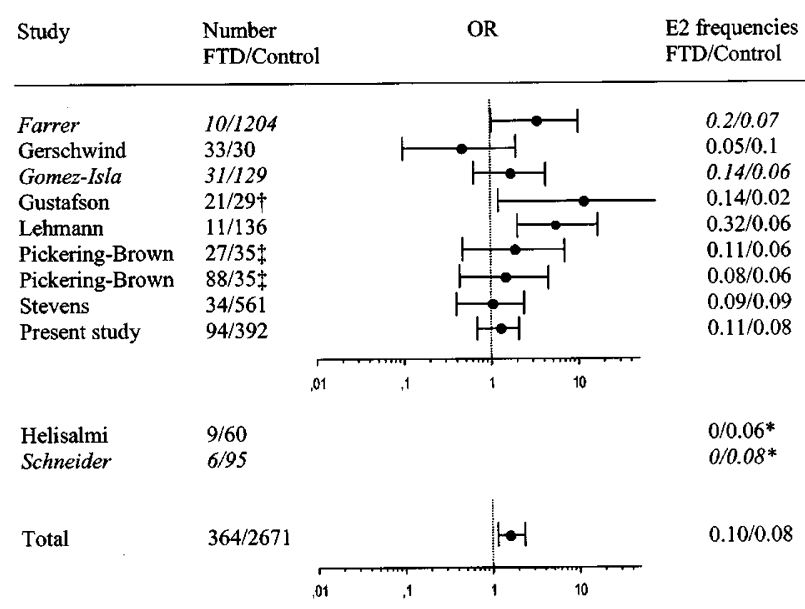

Figure 1 Meta-analysis of the E2 allele in FTD. MantelHaenszel statistics: $\chi^{2}=25.7,10 \mathrm{df}, P=0.004$. OR: odds ratios for the E2 allele vs E3 allele. Horizontal lines represent the $95 \% \mathrm{Cl}$ for the OR. †The OR $95 \% \mathrm{Cl}$ was $1.37-105.41$. $\$$ The control group was the same for the two studies and was therefore included only once in the pooled control group. *No OR estimation was possible because of division by 0 . The studies which were completely based on neuropathologically-confirmed patients are indicated in italics.

subgroup to the three others studies with neuropathological cases, and included the clinical-based subgroup of this study with the seven other clinical-based studies. In the studies with neuropathologically-confirmed FTD patients, we found a significant increase of the E2 allele frequency in patients (OR[E2 vs E3]=2.01; 95\%CI=1.02-3.98; $P=0.04)$. The same result was found in the clinical-based 
studies (OR[E2 vs $\mathrm{E} 3]=1.53 ;$ 95\%CI=1.06-2.23; $P=0.02)$. Moreover, heterogeneity disappeared in the neuropathological-based group (Mantel-Haenszel statistics: $P=n s$ ), but remained significant in the clinical-based group $(P=0.004)$.

The meta-analysis was also performed to test the role of the E4 allele. No significant difference was found (OR[E4 vs E3]=1.19; 95\% CI=0.92-1.54 including all studies; $\mathrm{OR}[\mathrm{E} 4$ vs E3]=1.20; 95\% $\mathrm{CI}=0.71-2.01$ in the neuropathologically-confirmed studies; OR[E4 vs E3]=1.19; 95\%CI=0.891.58 in the clinical-based studies).

\section{Discussion}

The results of our case-control study suggest that subjects who are homozygous for the APOE E2 allele are at increased risk for developing FTD. After stratification on familial history of FTD, the OR for E2E2 remains significant among patients with a positive $\mathrm{FH} \quad(\mathrm{OR}=23.8$; $P=0.019)$, even if the sample size is smaller (31 patients with FTD). Because E2 allele and E2E2 genotype frequencies are small in the general population (approximately $7 \%$ and $0.5 \%$ in the Caucasian population, respectively), our findings of a higher proportion of E2E2 genotype in patients compared to controls is marginally significant and predicated on very few subjects (three patients and one control). We have thus confirmed the genotype for these four subjects. Moreover, our data confirms some results of two previous studies. ${ }^{13,15}$ Indeed, Gustafson et al have found a significantly higher E2 frequency in their 21 patients with FTD (14.3\%) compared to 29 controls $(1.7 \%),{ }^{13}$ as did Lehmann et al ${ }^{15}$ who calculated an OR of 7.0 [2.5-19.5] for E2, using 11 patients with sporadic FTD (including nine patients with histopathological confirmation) and 136 controls from the cohort of the Oxford project to investigate memory and aging. In contrast, the implication of the E2 allele was not evidenced by a dozen studies which found either a significant increase of the E4 allele, or no significant difference in APOE allele or genotype frequency. These discrepancies could have many explanations. Firstly, the sample sizes of the previous studies were small (less then 35 except one study ${ }^{21}$ with 88 patients with FTD, in which two different subgroups are identifiable, the one of 49 autopsy verified patients and the other of 39 clinical-based patients). Most of them probably did not have sufficient a priori statistical power to determine the effect of the relatively rare E2 allele. Our study with 94 FTD patients was sufficiently powerful to detect an effect of equivalent size to that reported by Stevens et $a l^{14}$ for the E4 allele and by Lehmann et al ${ }^{15}$ for the E2 allele (80\% and >99\%, respectively). Secondly, most studies relied on clinical diagnosis without reference to valid criteria for FTD. In these studies, the inclusion of patients with misdiagnosed $\mathrm{AD}$ could occur as a possible confounding factor. Besides, all the studies which have found an association with the E4 allele were performed exclusively with clinical-based patients. The inadvertent inclusion of patients with $\mathrm{AD}$ would 'spuriously' inflate the E4 frequency and, on the contrary, decrease the E2 frequency. Since the Lund and Manchester consensus conference ${ }^{2}$, clinical criteria for FTD diagnosis are increasingly precise and allow to better distinguish between $\mathrm{AD}$ and FTD. If a significant number of patients with $\mathrm{AD}$ might have been inadvertently included in the first published studies, this has now become less likely. Although only a small proportion of the patients were examined neuropathologically (3\%) which allowed confirmation of the diagnosis of FTD, misdiagnosis as $\mathrm{AD}$ in our study is un-likely given the criteria used to assess patients. Indeed, diagnosis of FTD was based on strict criteria: (a) clinical diagnosis according to the LundManchester clinical consensus criteria for $\mathrm{FTD}^{2}$, (b) neuropsychological confirmation of frontal lobe dysfunction, (c) frontal or frontotemporal atrophy on computed tomography or magnetic resonance images, (d) frontal or frontotemporal hypoperfusion on single-photon emission computed tomographic images and, (e) clinical and neuropsychological annual follow-up. Thirdly, studies often provide little information on case-control matching. Bickeböller et al have demonstrated that the frequency of the E4 allele was lower in males over 60 years of age than in females. ${ }^{42}$ As the APOE allele frequency varies according to age and sex, it is crucial either to match patients and controls according to these two variables, or to take them into account by multivariate analyses.

In the meta-analysis, we examined seven case-control studies with genotype information ${ }^{14,15,18,20,22-24}$ and three more with allele information ${ }^{13,17,21}$ (total of 364 FTD patients and 2671 controls including ours). Using genotypes, the meta-analysis did not allow to reach statistically significant level even if the E2E2 genotype was more frequent in patients than in controls. We found a significant increase of the E2 allele frequency in FTD patients compared to controls, but also a heterogeneity between the different studies. We have then performed the metaanalysis in two more 'homogeneous' groups, based on the study design: on the one hand, the studies with available data in autopsy group of patients (neuropathologicallyconfirmed studies); on the other hand, the clinical-based studies. In the first group, an overall OR was then calculated which confirmed the role of the E2 allele in these patients with an accurate diagnosis of FTD. The same result was found in the clinical-based studies. Moreover, heterogeneity disappeared in the neuropathological-based group, but remained significant in the clinical-based group, showing the importance of the mode of recruitment for patients with FTD. We have also searched for an effect of the E4 allele in the meta-analysis. Any result was significant. Since we have found that the effect of APOE E2 is more marked in patients with positive family history, it would have been interesting to study the genotype and allele distributions according to $\mathrm{FH}$. Unfortunately, even if it was indicated 
in several previous studies, most of them did not provide enough information to stratify the meta-analysis on FH.

In summary, both the genetic association study and the meta-analysis indicate that the $\mathrm{E} 2$ allele may be a risk factor for FTD. However, these data should be interpreted with caution because of the relatively small sample size of patients with FTD analysed and the rarity of the E2E2 genotype. As already stated by Lehmann et al in their article, ${ }^{15}$ if this finding was confirmed, it could provide new insights to explore mechanisms of differential risk related to APOE in neurodegenerative diseases as AD and FTD.

\section{Acknowledgements}

This work was supported by a grant from the 'Fondation pour la Recherche Médicale' and from INSERM.

\section{References}

1 Jackson M, Lowe J: The new neuropathology of degenerative frontotemporal dementias. Acta Neuropathol 1996; 91: 127-134.

2 Clinical and neuropathological criteria for frontotemporal dementia. The Lund Manchester Groups. J Neurol Neurosurg Psychiatry 1994; 57: 416-418.

3 Mendez MF, Selwood A, Mastri AR, Frey WH: Pick's disease versus Alzheimer's disease: a comparison of clinical characteristics. Neurology 1993; 43: 289-292.

4 Gearing M, Mirra SS, Hedreen JC et al: The Consortium to Establish a Registry for Alzheimer's Disease (CERAD). Part X. Neuropathology confirmation of the clinical diagnosis of Alzheimer's disease. Neurology 1995; 45: 461-466.

5 Spillantini MG, Murrell JR, Goedert M et al: Mutation in the tau gene in familial multiple system tauopathy with presenile dementia. Proc Natl Acad Sci USA 1998; 95: 7737 -7741.

6 Hutton M, Lendon CL, Rizzu P et al: Association of missense and 5 '-splice-site mutations in tau with the inherited dementia FTDP17. Nature 1998; 393: $702-705$.

7 Poorkaj P, Bird TD, Wijsman E et al: Tau is a candidate gene for chromosome 17 frontotemporal dementia. Ann Neurol 1998; 43: $815-825$.

8 Dumanchin C, Camuzat A, Campion D et al: Segregation of a missense mutation in the microtubule-associated protein tau gene with familial frontotemporal dementia and parkinsonism. Hum Mol Genet 1998; 7: 1825 -1829.

9 Reed LA, Wszolek ZK, Hutton M: Phenotypic correlations in FTDP17. Neurobiol Aging 2001; 22: 89-107.

10 Miyamoto K, Kowalska A, Hasegawa M et al: Familial frontotemporal dementia and parkinsonism with a novel mutation at an intron 10+11-splice site in the tau gene. Ann Neurol 2001; 50: $117-120$.

11 Rizzu P, Van Swieten JC, Joosse M et al: High prevalence of mutations in the microtubule-associated protein tau in a population study of frontotemporal dementia in the Netherlands. Am J Hum Genet 1999; 64: 414-421.

12 Brown J, Ashworth A, Gydesen S et al: Familial non-specific dementia maps to chromosome 3. Hum Mol Genet 1995; 4: $1625-1628$.

13 Gustafson L, Abrahamson M, Grubb A, Nilsson K, Fex G: Apolipoprotein-E genotyping in Alzheimer's disease and frontotemporal dementia. Dement Geriatr Cogn Disord 1997; 8: 240-243.

14 Stevens M, van Duijn CM, de Knijff P et al: Apolipoprotein E gene and sporadic frontal lobe dementia. Neurology 1997; 48: 15261529.
15 Lehmann DJ, Smith AD, Combrinck M, Barnetson L, Joachim C: Apolipoprotein $\mathrm{E}$ epsilon2 may be a risk factor for sporadic frontotemporal dementia. J Neurol Neurosurg Psychiatry 2000; 69: $404-405$.

16 Czech C, Monning V, Tienari PJ et al: Apolipoprotein E-epsilon 4 allele and Alzheimer's disease. Lancet 1993; 342:1309.

17 Pickering-Brown SM, Siddons M, Mann DM et al: Apolipoprotein E allelic frequencies in patients with lobar atrophy. Neurosci Lett 1995; 188: 205-207.

18 Helisalmi S, Linnaranta $\mathrm{K}$, Lehtovirta $\mathrm{M}$ et al: Apolipoprotein $\mathrm{E}$ polymorphism in patients with different neurodegenerative disorders. Neurosci Lett 1996; 205: 61-64.

19 Minthon L, Hesse C, Sjogren M et al: The apolipoprotein E epsilon4 allele frequency is normal in fronto- temporal dementia, but correlates with age at onset of disease. Neurosci Lett 1997; 226: $65-67$.

20 Geschwind D, Karrim J, Nelson SF, Miller B: The apolipoprotein E epsilon4 allele is not a significant risk factor for frontotemporal dementia. Ann Neurol 1998; 44: 134-138.

21 Pickering-Brown SM, Owen F, Isaacs A et al: Apolipoprotein E epsilon4 allele has no effect on age at onset or duration of disease in cases of frontotemporal dementia with pick- or microvacuolartype histology. Exp Neurol 2000; 163: 452-456.

22 Farrer LA, Abraham CR, Volicer L et al: Allele epsilon 4 of apolipoprotein $\mathrm{E}$ shows a dose effect on age at onset of Pick disease. Exp Neurol 1995; 136: $162-170$.

23 Schneider JA, Gearing M, Robbins RS, de l'Aune W, Mirra SS: Apolipoprotein $\mathrm{E}$ genotype in diverse neurodegenerative disorders. Ann Neurol 1995; 38: 131-135.

24 Gomez-Isla T, West HL, Rebeck GW et al: Clinical and pathological correlates of apolipoprotein E epsilon 4 in Alzheimer's disease. Ann Neurol 1996; 39: 62-70.

25 Neary D, Pickering-Brown S, Roberts DA, Owen F: Apolipoprotein E4 alleles and Non-Alzheimer's Disease forms of dementia. Neurodegeneration 1993; 2: 300-301.

26 Fabre SF, Forsell C, Viitanen M et al: Clinic-based cases with frontotemporal dementia show increased cerebrospinal fluid tau and high apolipoprotein E epsilon4 frequency, but no tau gene mutations. Exp Neurol 2001; 168: 413-418.

27 Pickering-Brown SM, Mann DM, Bourke JP et al: Apolipoprotein E4 and Alzheimer's disease pathology in Lewy body disease and in other beta-amyloid-forming diseases. Lancet 1994; 343:1155.

28 Arai H, Higuchi S, Muramatsu T et al: Apolipoprotein E gene in diffuse Lewy body disease with or without co-existing Alzheimer's disease. Lancet 1994; 344:1307.

29 St Clair D, Norrman J, Perry R et al: Apolipoprotein E epsilon 4 allele frequency in patients with Lewy body dementia, Alzheimer's disease and age-matched controls. Neurosci Lett 1994; 176: $45-46$.

30 Frisoni GB, Geroldi C, Bianchetti A et al: Apolipoprotein E epsilon 4 allele frequency in vascular dementia and Alzheimer's disease. Stroke 1994; 25: $1703-1704$.

31 Noguchi S, Murakami K, Yamada N: Apolipoprotein E genotype Alzheimer's disease. Lancet 1993; 342: 737.

32 Anouti A, Schmidt K, Lyons KE et al: Normal distribution of apolipoprotein E alleles in progressive supranuclear palsy. Neurology 1996; 46: 1156-1157.

33 Arai H, Muramatsu T, Higuchi S, Sasaki H, Trojanowski JQ: Apolipoprotein E gene in Parkinson's disease with or without dementia. Lancet 1994; 344:889.

34 Marder K, Maestre G, Cote L et al: The apolipoprotein epsilon 4 allele in Parkinson's disease with and without dementia. Neurology 1994; 44: 1330-1331.

35 Folstein MF, Folstein SE, McHugh PR: 'Mini-mental state'. A practical method for grading the cognitive state of patients for the clinician. J Psychiatr Res 1975; 12: 189-198.

36 Mattis S: Dementia rating scale Odessa, FL: Psychological Assessment Ressources Inc 1988. 
37 Grober E, Buschke H: Genuine memory deficits in dementia. Dev Neuropsychol 1987; 3: 13-36.

38 Dubois B, Slachevsky A, Litvan I, Pillon B: The FAB: a Frontal Assessment Battery at bedside. Neurology 2000; 55: 1621-1626.

39 Hixson JE, Vernier DT: Restriction isotyping of human apolipoprotein $\mathrm{E}$ by gene amplification and cleavage with HhaI. J Lipid Res 1990; 31: $545-548$.
40 Mantel N, Haenszel W: Statistical aspects of the analysis of data from retrospective studies of disease. JNCI 1959; 22: 719-748.

41 Pignon JP, Poynard T: Meta-analysis of clinical trials. Gastroenterol Clin Biol 1991; 15: 229-238.

42 Bickeböller H, Campion D, Brice A et al: Apolipoprotein E and Alzheimer disease: genotype-specific risks by age and sex. Am J Hum Genet 1997; 60: 439-446. 